\title{
SPATIAL LOCALIZED SINGLE QUANTUM HETERONUCLEAR POLARIZATION TRANSFER
}

\author{
D.E. Demco And I. Ardelean
}

Technical University, Physics Department, 3400 Cluj-Napoca, Romania

(Received May 10, 1994)

\begin{abstract}
The existence of a strong spatial localization for the efficiency of cross-polarization by single quantum transition in the presence of a main field gradient was evidenced. The case of the solids with strong dipolar interactions has been analyzed. The slice profile of the transferred polarization was evaluated for different cross-polarization procedures. The spatial localization is superior as compared with the spin-locking slice selection method.
\end{abstract}

PACS numbers: 76.70.-r, 76.70.Fz, 33.35.-q

\section{Introduction}

Due to the spatial resolution of images ultimately limited by sensitivity, most NMR imaging experiments have been done on ${ }^{1}$ II. Ilowever in recent years an increase in NMR imaging techniques into the realm of solid materials has been observed.

The proton linewidth of solids is broadened by strong homonuclear dipolar interactions which has to be removed for an efficient spatial encoding by the magnetic field gradient. In this respect a variety of line-narrowing methods based on coherent averaging multiple pulse [1,2], magic angle sample spinning [3], magic angle narrowing in the rotating frame $[4,5]$ and combinations of them, has been considered [6]. Alternative approaches using RF field gradients have been suggested $[7,8]$. The NMIR images of solids can be obtained by using the high order multiple quantum spectra to increase the line separation due to applied gradient by a factor of $n$ equal to the number of Larmor frequency quantum characterizing the observed spectrum $[9,10]$.

Another class of techniques is based on Fourier spatial phase encoding using free induction decay [11], solid ccho [12, 13], Jeener-Brockaert pulse sequence $[13 ; 14]$, magic and rotary magic echoes [15]. NQR imaging of quadrupolar nuclei was also studied $[16,17]$.

The problem of severe homogeneous broadening due to homonuclear dipolar coupling can be eliminated by examining magnetically dilute spins in solids. In this 
respect NMR imaging of dilute $1 / 2$ spin ${ }^{13} \mathrm{C}$ isotope has been published [18-20]. The dilute nuclei heterogencous line narrowing is achieved by a combination of high-power proton decoupling combined in some cases with magic angle sample spinning and deconvolution. Of course, the sensitivity limitations due to natural abundance of ${ }^{13} \mathrm{C}$ in typical solids can be severe and hence the techniques that can enhance ${ }^{13} \mathrm{C}$ spin polarization are of interest. These are based on rotating frame heteronuclear polarization transfer using spin-locking procedure alone $[18,20]$ or in combination with ${ }^{1}$ II spin polarization via dynamic nuclear polarization [19].

Recently it has been pointed out [20] that the NMR images of rigid solids ${ }^{13} \mathrm{C}$ observation can yicld images of nearly the same quality as ${ }^{1} 1 \mathrm{l}$ observation. The small homogencous linewidth, high- $Q$ probes and narrow-band receivers associated with ${ }^{13} \mathrm{C}$ NMR compensate to a large extend for the low natural abundance nuclei. Additional chemical information can be gained by recording the NMIR imaging of dilute spins in solids.

Considering ${ }^{13} \mathrm{C}$ NMIR imaging of solids as a viable alternative to ${ }^{1}$ II NMIR imaging in solids, in this paper we intend to analyze the behaviour of polarization transfer methods used for increasing the dilute spin polarization operating in the presence of laboratory frame gradient. The spatial dependence of polarization transfer process on various experimental conditions could be exploited for NMR imaging and volume selective spectroscopy experiments.

We shall concentrate here on the case of strongly dipolar coupled spins in solids with abundant $I=1 / 2$ spins and dilute $S=1 / 2$ spins $\left({ }^{18} \mathrm{C},{ }^{15} \mathrm{~N},{ }^{29} \mathrm{Si}\right.$, ${ }^{31} \mathrm{P}$, etc.). In the following we will present the results which introduce a new dimension - the spatial one - in the ficld of heteronuclear polarization transfer dynamics. $\Lambda$ preliminary discussion of this spatial localization was presented in Refs. [21] and [22].

\section{The Hamiltonian and reference frame}

In this section we recall some details to the Ifamiltonian and quantum-meclanical interaction representation which occur for the polarization transfer in the rotating frame experiments $[23,24]$.

We discuss the situation only in which we may neglect the diffusion of the spins and spin-lattice relaxation times arc taken infinitely long.

The most common spin system encountered in the polarization transfer experiments contains two spin species $I$ and $S$ with different magnetogyric ratios $\gamma_{I}$ and $\gamma_{S}$. The sample contains $N_{I}$ and $N_{S}$ spins and in general $N_{I} \gg N_{S}$.

We shall consider in the following the experimental situation which is encountered in the laboratory frame NMR imaging experiment [25], i.c. the sample is placed in a large static homogencous magnctic ficld gradient $\boldsymbol{G}_{0}$. In order to simplify the problem but without loss of generality, the magnetic field $\boldsymbol{B}_{0}$ and magnetic field gradient $G_{0}$ will be applied along the $z$-axis. The case of rotating frame spatial encoding, using radiofrequency field gradients $[26,27]$ will be discuss elsewhere.

The high ficld polarization transfer IIamiltonian defined for a $z$-slice in the 
laboratory reference frame is

$$
I(z, t)=I I_{I}(z)+I_{S}(z)+I_{I S}(z)+I I_{\mathrm{RF}}(l) .
$$

The IIamiltonian for the abundant $I$ spin system is defined as

$$
I I_{I}(z)=I I_{I Z}(z)+I_{I I}(z) \text {, }
$$

where the Zeeman IIamiltonian is

$$
H_{I Z}(z)=-\left[\omega_{0 I}+\Delta \Omega_{I}(z)\right] I_{z}(z)
$$

with $\omega_{0 I}=\gamma_{I} B_{0}$ and $\Delta \Omega_{I}(z)=\gamma_{I} G_{0 z} z$. The $I$ spin operator is defined for $z$-plane as

$$
I(z)=I_{i} \int_{z-\text { slice }} \rho(r) \mathrm{d} x \mathrm{~d} y,
$$

where $I_{i}$ is the spin opcrator for an individual " $i$ " nucleus and $\rho(r)$ is the density operator. Without loss of gencrality we can include the contribution of the $I$-chemical-shift IIamiltonian in the Zeeman torm $I_{I Z}(z)$. The term $H_{I I}(z)$ describes the localized spin-spin interaction between $I$ spins. For polarization transfer experiments in solids the all sample dipolar IIamiltonians $H_{I I}=\sum_{z} H_{I I}(z)$ contribute in principle to the spin dynamics. If we consider a particular $z$-slice with macroscopic dimensions and the $r^{-3}$ spatial dependence of the dipolar interaction it is a good approximation to assume that the spin dynamics is determined only by the dipolar Iramiltonian for the $z$-slice.

The spatial localized IIamiltonian $I_{S}(z)$ which characterizes the dilute $S$ spins is

$$
I I_{S}(z)=I I_{S Z}(z)+I I_{S S}(z),
$$

where the terms have the same meaning as in Eq. (2).

The interaction $I I_{S S}(z)$ between the $S$ spins is ignored because of their low abundance.

The last term of Eq. (1) which describes interactions of the spin system with linearly polarized radiofrequency magnetic fields of amplitudes $2 B_{1 I}$ and $2 B_{1 S}$, and frequencies $\omega_{I}$ and $\omega_{S}$, respectively, has the form

$$
H_{\mathrm{RF}}(l)=-2 \omega_{1 I} I_{x} \cos \omega_{I} \ell-2 \omega_{1 S} S_{x} \cos \omega_{S} t
$$

where $\omega_{1 I}=\gamma_{I} B_{1 I}$ and $\omega_{1 S}=\gamma_{S} B_{1 S}$. The total spin operators are $I=\sum_{i} I_{i}$ and $S=\sum_{m} S_{m}$, respectively.

The adiabatic demagnetization in the rotating frame (ADRF) [23] can be taken into account by considering a continuous variation in the amplitude of the radiofrequency field.

The time evolution of the statistical cnsemble composed of many identical particles can be described by a density operator $\rho(l)$, which satisfies the Liouville-von Neuman cquation

$$
\mathrm{i} \frac{\partial \rho(t)}{\partial t}=\widehat{\Pi}(l) \rho(l)
$$

where we denoted a Liouville operator corresponding to an operator 0 by $\hat{0}$.

The total IIamiltonian and whole sample density operator can be written

$$
H(t)=\sum_{z_{\min }}^{z_{\max }} H(z, l)
$$


and

$$
\rho(t)=\sum_{z_{\min }}^{z_{\max }} \rho(z, l)
$$

where $z_{\min }$ and $z_{\max }$ definc the dimension of the sample along the $z$-axis.

With a good approximation the spin interaction between different $z$-slices (or volume elements) can be neglected and this together with a very slow spin difusion leads to the spatial localized spin temperature [28]. Within this limit we can write

$$
\left[I\left(z^{\prime}, t\right), I(z, t)\right]=0
$$

and

$$
\left[I I\left(z^{\prime}, t\right), \rho(z, \iota)\right]=0
$$

for $z \neq z^{\prime}$.

Ilence the Liouville-von Neuman equation can be written as a linear superposition of local density operator equations

$$
\mathrm{i} \frac{\partial \rho(z, t)}{\partial t}=\widehat{\Pi}(z, l) \rho(z, t) \text {. }
$$

The rotating frame heteronuclear polarization transfer is conveniently described in the double tilted rotating frames. In this quantum mechanical representation the local density operator evolves under the equation

$$
\mathrm{i} \frac{\partial \rho_{T R}(z, t)}{\partial t}=\widehat{\Pi}_{T R}(t) \rho_{T R}(z, t),
$$

where

$$
\rho_{T R}(z, t)=\widehat{T} \hat{R} \rho(z, t)
$$

with $R=R_{I} R_{S}, T=T_{I} T_{S}$ and

$$
\begin{aligned}
& R_{I}=\exp \left[-\mathrm{i} \omega_{I} I_{z}(z) t\right] ; R_{S}=\exp \left[-\mathrm{i} \omega_{S} S_{z}(z) t\right] ; \\
& T_{I}=\exp \left[\mathrm{i} 0_{I}(z) I_{y}(z)\right] ; T_{S}=\exp \left[\mathrm{i} 0_{S}(z) S_{y}(z)\right] .
\end{aligned}
$$

The local polar tilted angles are defined as

$$
o_{I}(z)=\tan ^{-1}\left[\frac{\omega_{1 I}}{\omega_{0 I}-\omega_{I}+\Delta \Omega_{I}(z)}\right]
$$

and respectively

$$
\theta_{S}(z)=\tan ^{-1}\left[\frac{\omega_{1 S}}{\omega_{0 S}-\omega_{S}+\Delta \Omega_{S}(z)}\right] .
$$

The relevant IIamiltonian in Eq. (12) is

$$
H_{T R}(z)=\widehat{T} \hat{R} I I+\mathrm{i}(T \dot{R})(T R)^{+} .
$$

Using Eqs. (1)-(5) and Eqs. (12)-(18), we finally obtain

$$
\begin{aligned}
& H_{T R}(z)=-\omega_{\mathrm{eft}, I}(z) I_{\bar{z}}(z)-\omega_{\mathrm{efr}, S}(z) S_{\bar{z}}(z) \\
& +P_{2}\left(\cos 0_{I}(z)\right) I_{I I}^{0}(z)+I_{p}(z) .
\end{aligned}
$$


In the above equations the rotating and tilted rotating frames axes are denoted by $(\mathrm{x}, \mathrm{y}, \mathrm{z})$ and $(\bar{x}, \bar{y}, \bar{z})$, respectively.

For the $z$-slice, the effective frequencics are defined as

$$
\begin{aligned}
& \omega_{\mathrm{eff}, I}(z)=\left[\omega_{1 I}^{2}+\left(\Delta \omega_{I}+\Delta \Omega_{I}(z)\right)^{2}\right]^{1 / 2}, \\
& \omega_{\mathrm{eff}, S}(z)=\left[\omega_{1 S}^{2}+\left(\Delta \omega_{S}+\Delta \Omega_{S}(z)\right)^{2}\right]^{1 / 2},
\end{aligned}
$$

where $\Delta \omega=\omega_{0}-\omega$ for $I$ and $S$ spin systems.

The localized $I-S$ dipolar coupling IIamiltonian, which can be considered as a perturbation, has the form

$$
\begin{array}{r}
I_{p}(z)=\cos 0_{I}(z) \cos \theta_{S}(z) \sum_{i, m}^{z-\text { slice }} b_{i m} I_{i \bar{z}} S_{m \bar{z}} \\
+\sin 0_{I}(z) \sin 0_{S}(z) \sum_{i, m}^{z-\text { slice }} b_{i m} I_{i \bar{x}} S_{m, \bar{x}} \\
-\sin 0_{I}(z) \cos 0_{S}(z) \sum_{i, m}^{z-\text { slice }} b_{i m} I_{i \bar{x}} S_{m \bar{z}} \\
-\cos 0_{I}(z) \sin 0_{S}(z) \sum_{i, m}^{z-\text { slice }} b_{i m} I_{i \bar{z}} S_{m \bar{x}}
\end{array}
$$

where $b_{i m}$ are the heteronuclear coupling constants.

\section{Spatial localized polarization transfer dynamics}

In order to analyze the heteronuclear polarization transfer dynamics [29-31] in a solid, subject to a main magnetic ficld gradient oriented in a particular $z$-direction, we introduce the $z$-slice observables. In our case these are represented by the thermodynamic coordinates defined as

$$
\left\langle Q_{i}(z)\right\rangle=\operatorname{Tr}\left\{Q_{i T R}(z) \rho_{T / R}(z, t)\right\},
$$

where $Q_{i, T R}(i=1,2)$ are the relevant quantum-mechanical operators for each of the systems involved in the polarization transfer process.

The spin dynamics can be analyzed in two extreme conditions. The first one corresponds to the spin-locking procedure (SL) [23]. In this case the spatial localized observables are represented by the llamiltonians

$$
\begin{aligned}
& Q_{1}(z)=-\omega_{\mathrm{eff}, I}(z) I_{\bar{z}}(z)+P_{2}\left(\cos 0_{I}(z)\right) I_{I I}(z), \\
& Q_{2}(z)=-\omega_{\mathrm{eff}, S}(z) S_{\bar{z}}(z) .
\end{aligned}
$$

These two spin subsystems are coupled by the Ilamiltonian $H_{p}(z)$ given be Eq. (21).

The second procedure corresponds to a low-eflective field condition for $I$ spins and it is achieved by adiabatic demagnetization in the rotating frame (ADRF) [23], or by a Jeener-Brockacrt pulse sequence [32]. Thus the observables of interest in the ADRF case are given by IIamiltonians

$$
Q_{1}(z)=H_{I I}^{0}(z), \quad Q_{2}(z)=-\omega_{\mathrm{efr}, S} S_{z}(z)
$$


and the coupling IIamiltonian, Eq. (21), can be obtained by considering that the tilt angle $0_{I}=0$.

We can define new $z$-slice thermodynamic coordinates

$$
\beta_{i}(z, t)=\left\langle Q_{i}(z)\right\rangle_{t} / \operatorname{Tr}\left\{Q_{i}^{2}(z)\right\} \quad(i=1,2)
$$

which in the high-temperature approximation have the dimensions of inverse spin temperature $\left(k_{\mathrm{B}}=1\right)$. The validity of the spatial localized spin temperature in solids has been discussed in Ref. [28].

Suppose now that we are interested in the polarization transfer dynamics of dilute spins [33]. In this case the following transport cquation for the local thermodynamic coordinate is valid

$$
\frac{\partial \beta_{2}(z, t)}{\partial t}=\frac{\beta_{1}(z, l)-\beta_{2}(z, t)}{T_{I S}(z)},
$$

where the spatial localized parameter, $T_{I S}^{-1}(z)$, characterizes the polarization transfer rate for the $z$-slice in the sample.

Equation (26) describes the dynamics of polarization transfer in the long range time domain, i.c. for $t \gg \tau_{\mathrm{c}}$, where $\tau_{\mathrm{c}}$ is the correlation time for the dipolar fluctuation of the $I$ spin system, but not in the time region of transient oscillations [29], for the strong dipolar coupled spins or in the case of solids with resolved dipolar structure $[34,35]$.

Within the limit of a very short corrclation time the spatial localized polarization transfer rate [29] for the SI, case is

$$
T_{I S}^{-1}=\frac{1}{2} \sin ^{2} \theta_{I}(z) \sin ^{2} \theta_{S}(z) M_{2, S I} J_{x}\left(\Delta \omega_{\mathrm{efr}}(z)\right) .
$$

In the case of $A D R F$ procedure [29] we lave

$$
T_{I S}^{-1}(z)=\sin ^{2} o_{S}(z) M_{2, S I}(z) J_{z}\left(\omega_{\mathrm{eff}, S}(z)\right),
$$

where $M_{2, S I}(z)$ is the local Van-Vleck second moment of the magnetic resonance line determined by the cross-coupling dipolar interaction. The spatial localized IIartmann-IIahn mismatch is $\Delta \omega_{\mathrm{efr}}(z)=\omega_{\mathrm{err}, S}(z)-\omega_{\mathrm{efr}, I}(z)$.

Equation (27) is valid in the high effective field for the SL case, i.e. $\omega_{\text {eff }, I}(z)$, $\omega_{\mathrm{eff}, S}(z) \gg \omega_{\mathrm{L} I}(z)$, where $\omega_{\mathrm{L} I}(z)$ is the rotating frame local field for the $z$-slice. It is also valid only for $0_{I}(z)$ values different from the "magic angle" $0_{m I}=\cos ^{-1}\left(3^{-1 / 2}\right)$ for which the memory effect in the thermal bath becomes important in the case of dipolar interactions. For this "magic $z$-slice" which could occur in the sample as a result of the interplay between the values of magnetic ficld gradient, off-resonance set, RF field strength and the dimension of the sample, a coherent exchange of $I$ and $S$ magnetization takes place.

The spectral density functions which occur in the expressions of the polarization transfer rates, (Eqs. (27) and (28)), are given by

$$
\begin{aligned}
& J_{x}(\omega)=\int_{0}^{\infty} \mathrm{d} \tau \cos (\omega \tau) C_{x}(\tau), \\
& J_{z}(\omega)=\int_{0}^{\infty} \mathrm{d} \tau \cos (\omega \tau) C_{z}(\tau),
\end{aligned}
$$


where $C_{x}(\tau)$ and $C_{z}(\tau)$ are the dipolar fluctuation normalized autocorrelation functions [29]. It has been proved [29] that for small values of time the autocorrelation functions $C_{x}(\tau)$ and $C_{z}(\tau)$ have a Gaussian $[31,36]$ and a Lorentzian $[30,37,38]$ character,

$$
C_{x}(\tau) \approx \exp \left\{-\tau^{2} / \tau_{\mathrm{c}}^{2}\right\}
$$

and respectively

$$
C_{z}(\tau) \approx \frac{1}{1+\tau^{2} / \tau_{\mathrm{c}}^{2}}
$$

The correlation time is taken to be $\tau_{c}=\left(2 / M_{2}\right)^{1 / 2}$, where $M_{2}$ is the second moment of the corresponding spectral density function which can be expressed as a function of lattice sums [29]. This dipolar correlation time has been evaluated for some crystals [29,37,39] and powders [30,38].

From Eqs. (27)-(31) for the localized polarization transfer rate we obtain the following approximate expressions for the SL, procedure:

$$
T_{I S}(z)^{-1} \approx \frac{\sqrt{\pi}}{4} \sin ^{2} \theta_{I}(z) \sin ^{2} \theta_{S}(z) M_{2, S I}(z) \tau_{\mathrm{c}} \operatorname{cxp}\left[-\frac{\Delta \omega_{\mathrm{efl}}(z) \tau_{\mathrm{c}}^{2}}{4}\right]
$$

and for ADRF

$$
T_{I S}(z)^{-1} \approx \frac{\pi}{2} \sin ^{2} 0_{S}(z) M_{2, S I}(z) \tau_{\mathrm{c}} \exp \left[-\omega_{\mathrm{eff}, S}(z) \tau_{\mathrm{c}}\right] .
$$

We can define the normalized spatial localized polarization transfer rate by $r=T_{I S}^{-1}(z) / T_{I S}^{-1}(0)$. If the sample is homogencous, i.e. $M_{2, S I}(z)=M_{2, S I}(0)$ for any $z$-slice and the RF fields are applied on resonance, the $r$-functions have the form

$$
r=\sin ^{2} \sigma_{I}(z) \sin ^{2} \sigma_{S}(z) \exp \left\{-\frac{\left[\Delta \omega_{\mathrm{eff}}^{2}(z)-\left(\omega_{1 I}-\omega_{1 S}\right)^{2}\right]}{4} \tau_{\mathrm{c}}^{2}\right\}
$$

for SL and

$$
r=\sin ^{2} 0_{S}(z) \exp \left\{\left[\omega_{\mathrm{efr}, S}(z)-\omega_{1 S}(z)\right] \tau_{\mathrm{c}}\right\}
$$

for ADRF, respectively.

The normalized polarization transfer rate for ADRF, Eq. (35), is valid for any position in a solid with strong nonlocalized dipolar interactions. Equation (34), which describes the SI procedure, is not valid at the "magic position" or "magic slice" $Z_{\mathrm{m}}=B_{1 I} /\left(G_{0 z} \tan \theta_{\mathrm{m} I}\right)$ for which the transfer process is coherent [35].

To evaluate the inverse spin temperature spatial distribution for the $S$ spins we have to consider the local coupled rate equations which can be written in a matrix form

$$
\frac{\partial \beta(z, t)}{\partial t}=-\Lambda(z) \beta(z, l)
$$

where any spin-lattice relaxation processes were neglected and

$$
\begin{aligned}
& \beta(z, t)=\left[\begin{array}{l}
\beta_{1}(z, t) \\
\beta_{2}(z, t)
\end{array}\right], \\
& \Lambda(z)=\left[\begin{array}{cc}
\varepsilon_{\mathrm{a}}(z) T_{I S}^{-1}(z) & -\varepsilon_{\mathrm{a}}(z) T_{I S}^{-1}(z) \\
-T_{I S}^{-1}(z) & T_{J S}^{-1}(z)
\end{array}\right] .
\end{aligned}
$$


In Eq. (38), the ratio between the local heat capacities of $S$ and $I$ spins [23] was noted by $\varepsilon_{\mathrm{a}}(z)$ with $\mathrm{a}=\mathrm{SL}$ or $\mathrm{a}=\mathrm{ADRF}$.

The time evolution of the inverse effective spin temperature for the $S$ spins can be obtained from Eqs. (36)-(38)

$$
\beta_{2}(z, t)=\beta_{f}(z)\left\{1-\exp \left[-t / T_{I S}(z)\right]\right\},
$$

where $\beta_{f}(z)$ is the localized inverse spin-temperature reached by both spin systems. At the beginning of the contact period $\beta_{2}(z, 0)=0$.

\section{Spatial localized transferred magnetization}

During the hetcronuclear polarization transfer a Zeeman order is created in the tilted rotating frame of $S$ spins. The longitudinal local $S$-magnetization produced at the moment $t$ along the eflective field is

$$
M_{S}(z, l)=\frac{1}{3} \gamma_{S} S(S+1) \beta_{2}(z, l) N_{S}(z) \omega_{\text {erf }, S}(z)
$$

with the inverse spin temperalure $\beta_{2}(z, l)$ given by Eq. (39).

The detected instantaneous $S$-spin magnetization is

$$
M_{S}(z, t)_{\mathrm{det}}=M_{S}(z, t) \sin 0_{S}(z) \text {. }
$$

To evaluate the transferred magnetization we have to consider the spatial localized thermodynamics of the process. The localized equilibrium inverse temperature for both spin systems $\beta_{f}(z)$ is given by the following relation [23]:

$$
\beta_{f}(z)=\frac{\beta_{I, \mathrm{a}}(z, 0)}{\left(1+\varepsilon_{\mathrm{a}}(z)\right)},
$$

where $\beta_{I, \mathrm{a}}(z, 0)$ is the local inverse spin tempcrature of $I$ spins at the beginning of polarization transfer process.

We shall consider that the experiment was conducted so that a spin-lock pulse sequence was applied for the slice selection [40-42], bcfore the polarization transfer process begins. In Figs. 1a and $\mathrm{c}$ we represent the pulse sequences which use the slice selection by spin-locking applied previously to the SI and ADRF procedures. Also another possibility is presented in Fig. $1 \mathrm{~b}$. In this case no slice selective single or composite pulscs are applied before the ADRF cooling procedure.

The spatial profile of inverse spin temperature after a spin-lock pulse sequence is $[28]$ as follows:

$$
\beta_{I, S L}(z, 0)=\beta_{\mathrm{L}} \omega_{0} \omega_{1 I} / \omega_{\mathrm{eff}, I}^{2}(z),
$$

where $\beta_{\mathrm{L}}=1 /\left(k_{\mathrm{B}} T_{\mathrm{L}}\right), T_{\mathrm{L}}$ is the lattice temperature. $\Lambda \pi / 2$-delta preparation pulse was considered. The normalized spatial distribution of the spin-locking cooling efficiency is described by the Lorentzian function [28]

$$
s(z)=\frac{\beta_{I, S L}(z, 0)}{\beta_{I, S L}(0,0)}=\frac{1}{1+\left(G_{0 z} z / B_{1 I}\right)^{2}},
$$

where we suppose that the strength of RF field is bigger than the local field.

For the ADRF procedure (cf. Fig. 1b) we can write

$$
\beta_{I, \operatorname{ADRF}}(z, 0)=\beta_{\mathrm{L}} \omega_{0} / \omega_{\mathrm{L}, I}(z)
$$

an expression derived using entropy invariance [43]. 

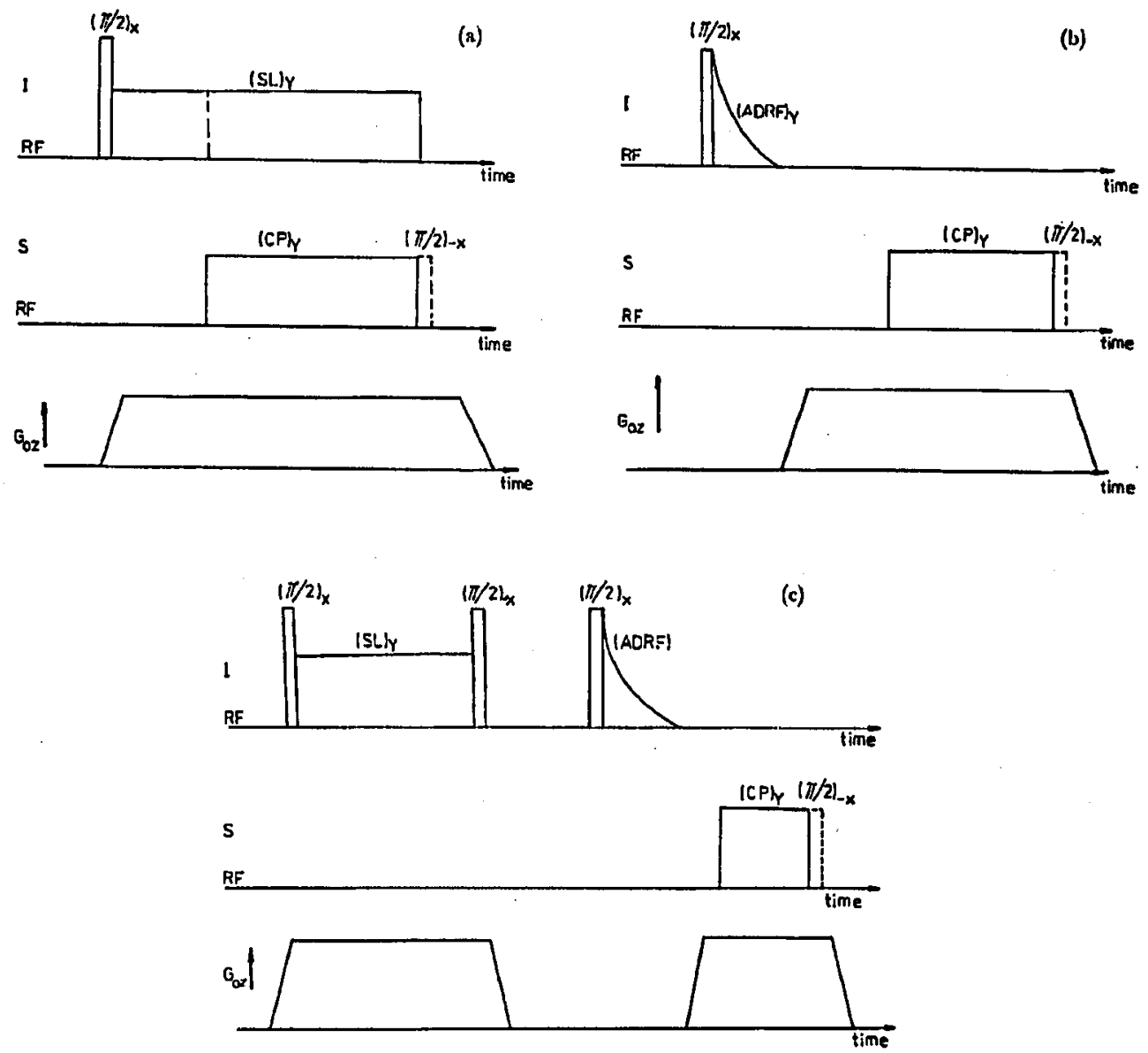

Fig. 1. A scliematic representation of the heteronuclear polarization transfer in solids using the SL and ADRF procedures applied in the presence of a constant main magnetic field gradient. In (a) the slice selection together with the spin-locking pulse sequence was used before the polarization transfer begins. In (b) the ficld gradient is applied after the dipolar order was created by the ADRF procedure. The presence of the gradient does not aftect the dipolar order. In (c) the slice selection together with the spin-locking pulse sequence was performed before the ADRF procedure was applied in the absence of the field gradient. This pulse sequence also includes the Zeeman-storage $(\pi / 2)-x$ pulses for $I$ and $S$-magnetization. Such an optional $S$-storage pulse can be used for (a) and (b) cases.

If the slice selection using the spin-lock pulse sequence is applied in combination with the $A D R F$ procedure from Eqs. (43) and (45) we get

$$
\beta_{I, \mathrm{ADRF}}^{\prime}(z, 0)=\beta_{I, \mathrm{SL}}(z, 0) \beta_{I, \mathrm{ADRF}}(z, 0) .
$$

The above equation is only approximate valid because, in general, Zeeman-storage $(\pi / 2)_{-x}$-pulse, (cf. Fig. 1c), distorts the profile of magnetization produced 
by SL-pulse sequence. To simplify the discussion, this distortion will be neglected. The local normalized $S$-spin detected signal for on-resonance excitation is defined by the following equation:

$$
s_{z}=\frac{M_{S}(z, t)_{\mathrm{det}}}{M I_{S}(0, l)_{\operatorname{det}}}
$$

From Eqs. (40) and (41) for the spatial profile of the transforred $S$-spin normalized magnetization we obtain

$$
s_{z}=\frac{\beta_{2}(z, l)}{\beta_{2}(0, l)} \frac{N_{S}(z)}{N_{S}(0)} .
$$

If $\varepsilon_{\mathrm{a}}(z)<1$ and from Eqs. (39), (10) and (17) we can write for the SL case (cf. Fig. 1a)

$$
s_{z}=\frac{\omega_{1 I}^{2}}{\left(\omega_{\mathrm{eff}, I}(z)\right)^{2}} \frac{1-\exp \left(-t / T_{I S}(z)\right)}{1-\exp \left(-l / T_{I S}(0)\right)} .
$$

The above expression is also valid for the $\Lambda$ DRF procedure combined with the spin-locking slice selection (cf. Fig. 1c), if we consider an lomogeneous sample again for which $\omega_{\mathrm{L} I}(z)=\omega_{\mathrm{L} I}(0)$. Of course, the functional dependence of signal profile on $z$ will be different compared with the SL case, as a result of different position dependence of polarization transfer ratc. For $t \gg T_{I S}(z)$, the signal profile is the same for the SL and ADRF procedures and coincide with the spin-locking slice selected profile.

For the procedure presented in Fig. 1b, the $S$-magnetization profile can be obtained from Eqs. (15) and (46). For an homogeneous sample we have

$$
S_{z}=\frac{1-\operatorname{cxp}\left(-t / T_{I S}(z)\right)}{1-\exp \left(-t / T_{I S}(0)\right)}
$$

The spatial selectivity of the polarization transfer is related directly to the position dependence of the polarization transfer rate vanishes in the limit of a very long contact time.

In the following we shall analyze the case when the rotation properties of $\pi / 2$-flip-back pulse $(F$-pulse) is affected by the presence of the gradient. It is easy to show [28] that the stored magnetization is related to the magnetization produced by the polarization transfer process by the relation

$$
M_{S}(z, t)_{\text {stored }}=F(z) M_{S}(z, t),
$$

where "flip"-function $F(z)$ is

$$
\begin{aligned}
F(z) & =\sin \theta_{S}(z) \sin \theta_{S}^{(F)}(z) \sin \left[\omega_{\mathrm{efr}, S}^{\left(F^{\prime}\right)}(z) \tau\right] \\
& +\cos 0_{S}(z) \sin ^{2} \theta_{S}^{(F)}(z) \cos \left[\omega_{\mathrm{err}, S}^{\left(F^{\prime}\right)}(z) \tau\right]+\cos 0_{S}(z) \cos ^{2} \theta_{S}^{(F)}(z) .
\end{aligned}
$$

The polar tilt angle $0_{S}^{(F)}(z)$ and the effective-frequency $\omega_{\text {efr }, S}^{(F)}(z)$ for the $F$-pulse are given by the same relations introduced before for the polarization transfer pulses (Eqs. (17) and (20)). The $F$-pulse is applicd on-resonance, i.e. $\theta_{S}^{(F)}(0)=\pi / 2$ and has a $\pi / 2$-rotation angle for the slice $z=0$, i.e. $\omega_{\mathrm{eff}, S}^{(F)}(0) \tau=$ $\omega_{1 S}^{(F)} r=\pi / 2, \tau-$ bcing the pulse duration. 
The normalized Zeeman-stored spatial dependent magnetization can be defined by the expression

$$
s_{z z}=\frac{M_{S}(z, l)_{\text {stored }}}{M_{S}(0, t)_{\text {stored }}} .
$$

Using Eqs. (41) and (47) in Eq. (53) we obtain finally

$$
s_{z z}=F(z) \frac{\omega_{\mathrm{ef}, S}(z)}{\omega_{1 S}} s_{z} .
$$

\section{The profile and width of the excited slice}

Using the computer simulations we intend to analyze the spatial distribution of the polarization transfer rates, the detected signals and the Zeeman-stored magnetization. We shall compare the spatial selectivity with that of the spin-lock composite pulse scquence. The on-resonance excitation for both spin species is suppose to be fulfilled.

\section{TABLE}

The dipolar spin corrclation times $\tau_{c}$ used for polarization rate evaluation in the case of the SL and ADRF procedurcs.

\begin{tabular}{l|c|c}
\hline \hline \multirow{2}{*}{ Sample } & \multicolumn{2}{|c}{$\tau_{\mathrm{c}}[\mu s]$} \\
\cline { 2 - 3 } & $\mathrm{SL}$ & $\mathrm{ADRF}$ \\
\hline $\mathrm{CaF}_{2}\left(B_{0} \|[111]\right)$ & $102[29]$ & $81[29]$ \\
Adamantane & $140[38]$ & $122[37,38]$
\end{tabular}

In the case of spatial cncoding using main magnetic field gradient the normalized transfer rates for $\mathrm{CaF}_{2}$ crystal with orientation $\mathrm{B}_{0} \|$ [111] and adamantane powder $\left(\mathrm{C}_{10} \mathrm{II}_{16}\right)$ (where ${ }^{43} \mathrm{Ca}$ and $13 \mathrm{C}=S,{ }^{19} \mathrm{~F}$ and ${ }^{1} \mathrm{II}=I$, respectively) were evaluated using the valucs of dipolar correlation times given in Table. The spatial dependence of the r-functions, Eqs. (34) and (35), for these two model samples in the case of the SL and ADRF procedures were represented in Figs. 2a-d for different values of mismatch IIartmann-IIalın (IIII) eflective frequency $\Delta \nu_{\text {eff }}$ and effective frequencies of $S$-polarization contact pulse.

We remark that the polarization transfer rates have a strong spatial dependence. Thie profiles are different for the SL and $\triangle D R F$ cases as a result of different functional spatial dependence evidenced by Eqs. (34) and (35). The profiles are in general non-rectangular but they are close to this shape in some special cases (see Fig. $2 \mathrm{c}$ for $\Delta \nu_{\text {efr }}=0 \mathrm{kHz}$ ). The profiles depend on the sample structure.

For $\mathrm{CaF}_{2}$ crystal the dipolar correlation time depends on the crystal orientation $[29,39]$. For instance we liave the computed ratios $\tau_{\mathrm{c}}\left([111] / \tau_{\mathrm{c}}\left(\left[\mathrm{b}_{\mathrm{c}} 00\right]\right)=2.76\right.$ for SL-case and $\tau_{\mathrm{c}}([111]) / \tau_{\mathrm{c}}([100])=1.80$ for ADRF. We expect (see Eqs. (34) 

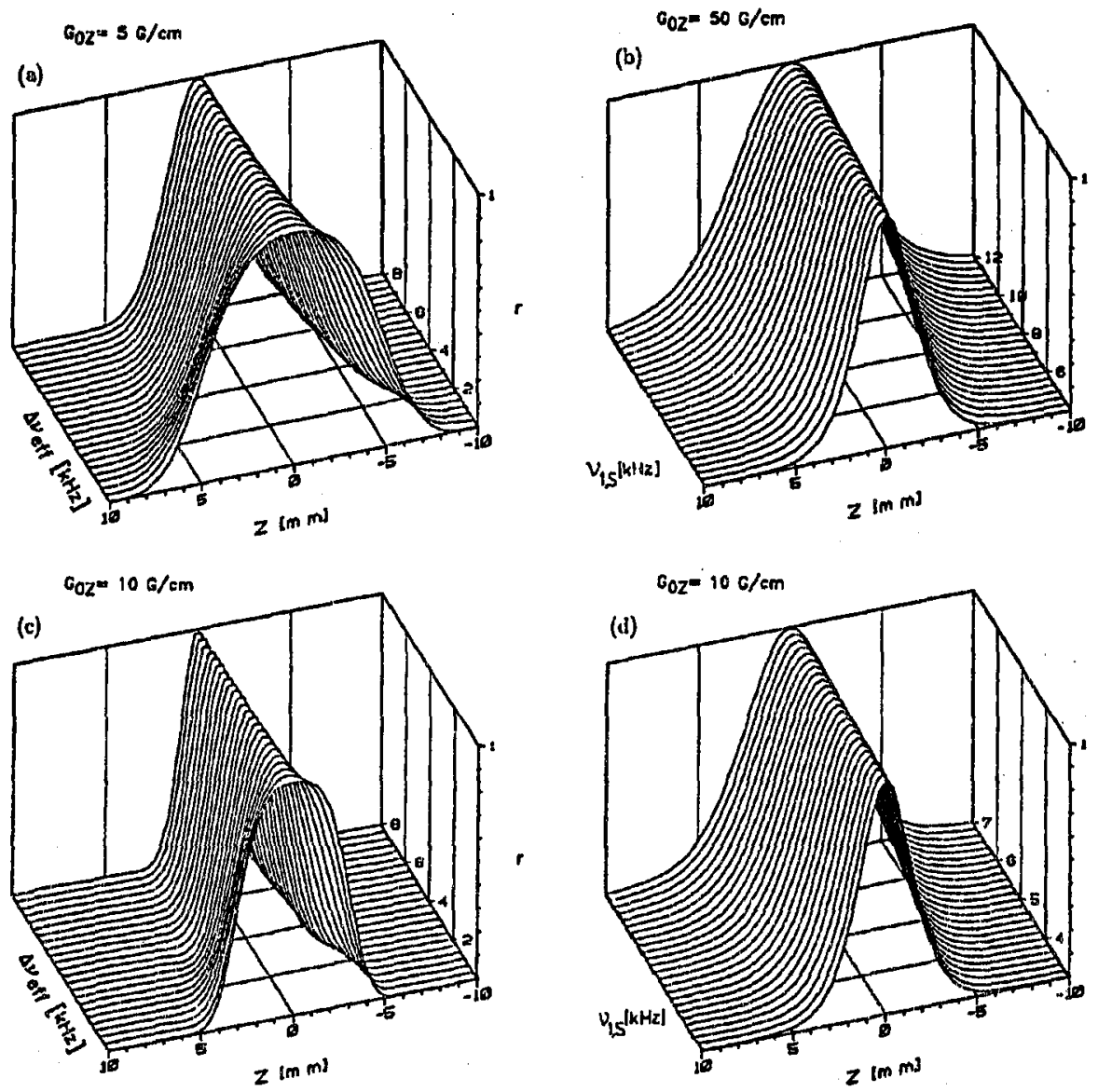

Fig. 2. The spatial dependence of the normalized polarization transfer rates $r$ for $\mathrm{CaF}_{2}$ crystal with orientalion $B_{0} \|$ [111] ((a) for SL and (b) for ADRF) and powdery adamantane ((c) for SL and (d) for ADRF) as a function of HH mismatch parameter $\Delta \nu_{\mathrm{eff}}$ and polarization transfer $S$-pulse strength $\nu_{1} s$. The magic slices are presented in Fig. 2c.

and (35)) a stronger spatial dependence of the normalized polarization transfer rate for SL compared with the ADRF procedure, if the crystal orientations are modified.

The magic-slice positions are given by the equation $Z_{\mathrm{m}}= \pm B_{1 J} /\left(2^{1 / 2} G_{0 z}\right)$ and are present only for the SL procedure. The $r$-functions are not valid at these positions.

In Fig. 2a the magic slices occur at the coordinates $Z_{\mathrm{m}}= \pm 14.14 \mathrm{~mm}$, and are out of the supposed sample spatial range. For stronger $G_{0 z}$ gradient the magic slices occur inside the sample (see Fig. 2c). For all these cases $B_{1 f}=10 \mathrm{G}$.

The half-width at the half-intensity for the normalized polarization transfer rates profiles versus the main field gradient strength is presented in Figs. 3a and $b$ for the SL and ADRF procedure, respectively. 

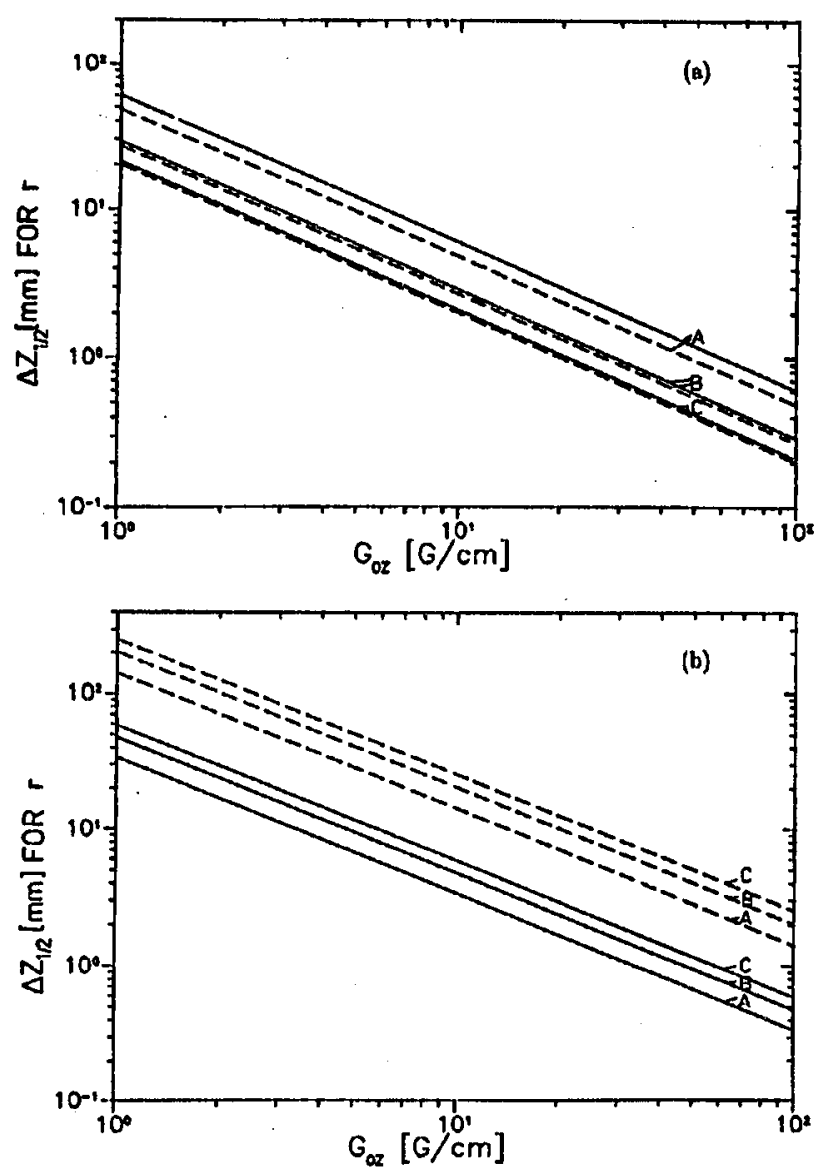

Fig. 3. The half-width at the half-intensity of $r$ function versus the gradient strength $G_{0 z}$, for SL (a) and $A D R F(b)$. The solid lines correspond to adamantane and dashed lines to $\mathrm{CaF}_{2}$ crystal. For curves A, B, C the Ilartmann-IIahn mismatch is $\Delta \nu_{\text {eft }}=0$, 4 and $8 \mathrm{kIIz}$ and $\nu_{1} s=3,5$ and $7 \mathrm{kIIz}$, respectively.

We can see that the polarization transfer process becomes strongly localized with increasing gradient strength. In the case of the SL procedure the localized efficiency of polarization transfer is only a little higher for $\mathrm{CaF}_{2}$ crystal compared with adamantane but it is vice versa for the ADRF procedure.

The normalized spatial distributed $S$-magnetization generated as a result of the polarization transfer process was represented in Figs. $4 a$ and $b$ for the SL and ADRF procedures as a function of IIII mismatch and the $S$-pulse strength. A contact transfer pulse of duration $t=N T_{I S}(0)$ with $N=3$, was considered. The profiles of transferred transverse magnetization are more rectangular compared with the profiles of the transfer rates. At the position of magic slices the function $s_{z}$ does not describe the transferred polarization for the SL case. 

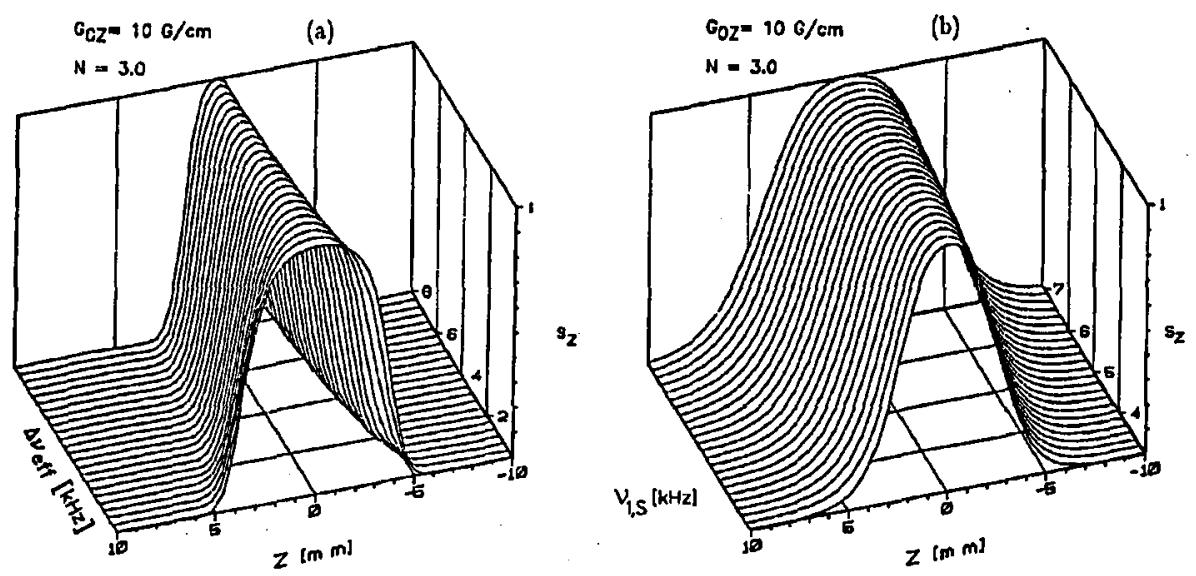

Fig. 4. The spatial dependence of the normalized $S$-spin polarization transfer detected magnetization $s_{z}$ for powdered adamantane in the case of the SL (a) and ADRF (b) procedures for different IIII mismatch $\Delta \nu_{\text {eff }}$ and polarization transfer pulse strength $\nu_{1} s$. The magic slices occur at the position $z_{\mathrm{n}}= \pm 7.07 \mathrm{~mm}$.

The half-width at the half-intensity of profiles $s_{z}$ versus the gradient strength was represented in Fig. 5 a together with $\Delta z_{1 / 2}$ value of the slice selection produced by the spin-lock pulse sequence with a RF field of $B_{1 I}=10 \mathrm{G}$.

We can see that for all experimental conditions the spatial regions, in which $S$-spin magnetization is generated, are narrower compared with the regions excited by the spin-lock pulse sequence. The most efficient procedure in this respect is SL, with the higher value of IIII mismatch. The most efficient ADRF procedure corresponds to one with smaller value of $S$ contact pulse strength.

When we discuss the spatial efficiency of transfer magnetization we have to consider the fact that the maximum transfer rate corresponds to the matched IIII condition (i.e. $\Delta \nu_{\mathrm{eff}}=0$ ) and at the same time the maximum magnetization transfer occurs when the $I$ and $S$ spin rescrvoirs have the same heat capacity [23]. These two conditions camot be fulfilled simultaneously.

If an on-resonance flip-back pulse with the same strength as the polarization transfer $S$-pulse is applied to store the $S$-spin magnetization along the $\boldsymbol{B}_{0}$ field the spatial dependent stored polarization is described by function $s_{z z}$ (see Eq. (54)). The half-width at the lialf-intensity of this stored polarization is represented in Fig. 5b.

The profile of stored magnetization is in general different from the transferred magnetization profile as a result of interplay of two factors: (i) the effect of gradient on the rotation properties of the flip-back pulse and (ii) the spatial encoded polar tilt angle of transferred magnetization. The comparison of Figs. 5a and 5b shows that these effects are not essential for our specified conditions. Consequently, the stored spatially distributed magnetization reproduces in a good approximation the spatially transferred magnetization behaviour.

The spatial selectivity of the polarization transfer is improved if we sacrifice 

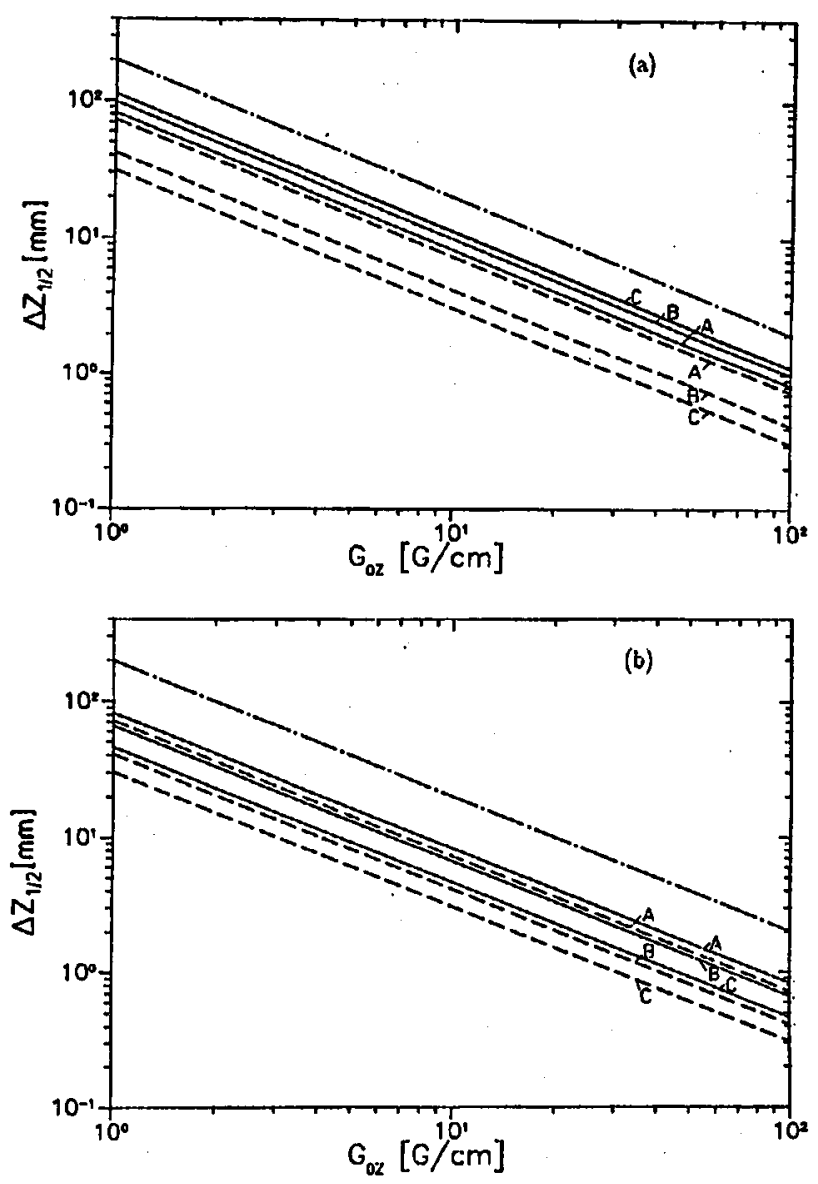

Fig. 5. The half-width at the half-intensity of $s_{z}$ versus gradient strength for SL-case (dashed line) and ADRF-case (continuous line) was represented in (a). The same representation (b) was done for $s_{z z}$-stored magnetization function. For all the curves the contact time was $t=N T_{I S}(0)$ with $N=3$. In the figures (a) and (b) $\Delta \nu_{\text {eff }}=0,4,8 \mathrm{kHz}$ and $\nu_{1 S}=3,5,7 \mathrm{kII} z$ correspond to curves A, B, C, respectively. Also the half-width at the half-intensity of the slice selection function $s(z)$ was represented (dashed-point line) in (a) and (b) for a lock-field strength of $B_{1 I}=10 \mathrm{G}$.

a part of produced $S$-spin magnetization by reducing the contact time between $I$ and $S$ spins. In such condition the transfer magnctization around the $z=0$ slice, for which the transfer rate is maximum, does not have time to occur.

We can see from Fig. 6a that the sclected slice by the SL polarization transfer process is approximately one order of magnitude smaller than the excited slice produced by the spin-lock pulse sequence. Even if the transfer magnetization profile is not rectangular, the corresponding region is well defined as compared with the 

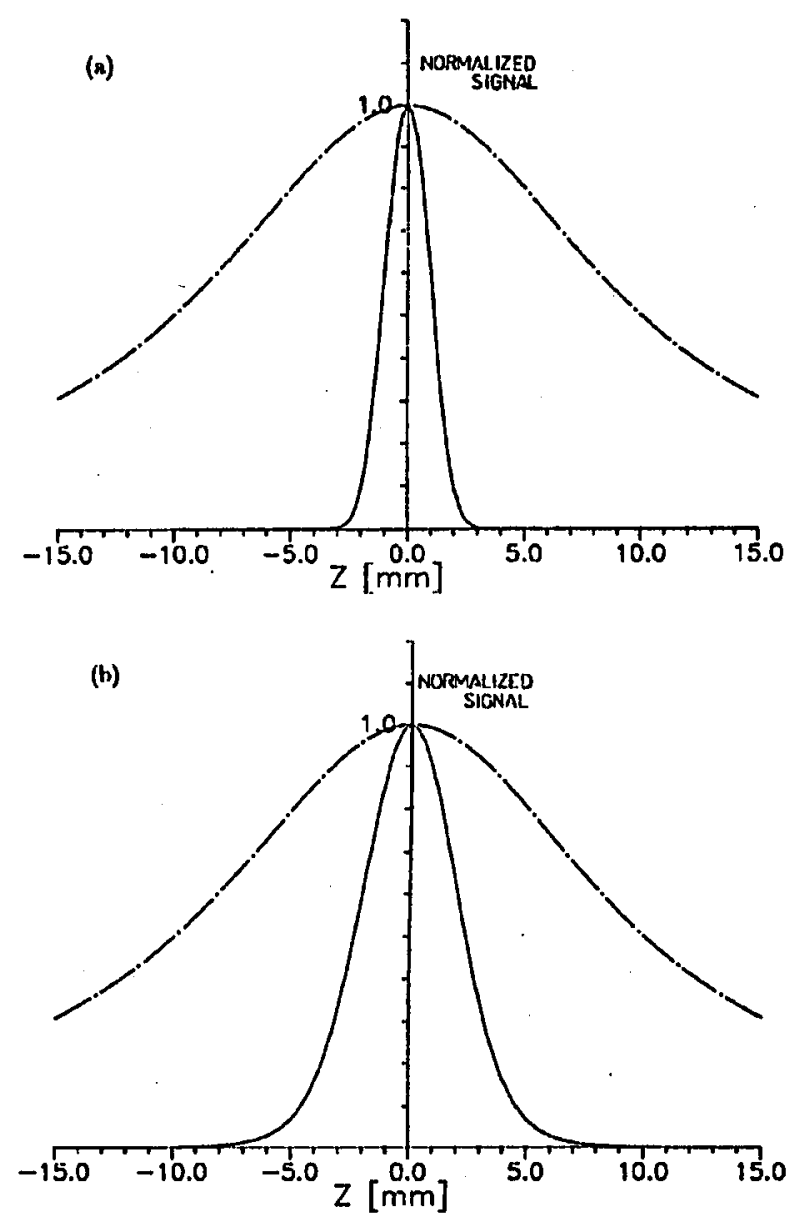

Fig. 6. The spatial distribution of the normalized $S$-spin detected magnetization (solid lines) for SL (a) and ADRF (b) polarization transfer experiment in which a slice was selected before the magnetization transfer (sce Figs. 1a and c). $\Lambda$ contact time $t=$ $N T_{I S}(0)$ for $N=3.0$ with $\Delta \nu_{\mathrm{eff}}=8 \mathrm{kII} / \mathrm{and} \nu_{1 S}=5 \mathrm{kIIz}$ was used. The spintlock slice profile is also represented (dashed-point line) for spin-lock pulse strength $B_{1 I}=10 \mathrm{G}$. The gradient strength is $G_{0 z}=10 \mathrm{G} / \mathrm{cm}$.

Lorentzian bchaviour of the excitation profile produced by the spin-lock pulse sequence [28]. We consider in these representations that $B_{1 I}=10 \mathrm{G}$ which is bigger than the rotating frame local field in adamantane $\left(B_{\mathrm{L}}=0.55 \mathrm{G}\right)$.

The same general bchaviour takes place in the case of ADRF polarization transfer but the produced slices are few times larger compared with the width produced by the SL polarization transfer. 'This is evident from Fig. $6 \mathrm{~b}$. In this figure we represent the normalized spatial dependent detected transfer magnetization 
function $s_{z^{\prime}}=s(z) s_{z}$, where $s(z)$ is the spatial profile of the sclected slice produced by the SL-pulse sequence (Eq. (14)) and $s_{z}$ is the spatial profile of the ADRF coherence transfer process (Eq. (50)). The slice profile described by the function $s_{z^{\prime}}$ corresponds to experimental strategies represented in Fig. 1c.

\section{Conclusions}

Ileteronuclcar polarization transfer in the rotating frame represents a viable method for spatial localization, which can be naturally integrated in the pulse sequences for imaging and volume selective spectroscopy of solids. If we consider a volume selective experiment three different types of strategy could be imagined:

(i) The transverse polarization or dipolar order of abundant $I$-spins can be produced in a localized volume using narrow band excitation pulse sequence applied in the presence of magnetic field gradients. This selected region with lower $I$-spin temperature can be used to transfer magnetization to rare $S$-spins in the same region. The polarization transfer process takes place in the absence of the field gradient.

(ii) At the begimning of the experiment the magnetization is transferred to the dilute $S$-spins uniformly in the sample. The localized region for $S$-spin magnetization can be selected using the "liquid state"-procedures in the presence of abundant spin decoupling.

(iii) The transfer of polarization and the spatially selective excitation can be performed in an integrated manner. In this latter case it is essenitial to understand the polarization transfer dynamics in the prescnce of a field gradient. This method has the advantage of lower RF power dissipation and possibility of performing a much more efficient slice and finally volume sclection.

A detailed theorctical analysis and computer experiments wore performed for crystalline and powder samples for diflerent cooling procedures of abundant spins.

The polarization transfer rates show a strong spatial dependence which is mainly related to the spatial encoded Hartmann-IJahn mismatch. This spatial dependence is very strong only for a limited contact time beiween spin systems. The profile of the transferred $S$-magnctization is not rectangular but the excitation is well localized as compared for instance with the homonuclear spin-locking pulse sequence [28].

The polarization transfer in a lieteronuclear rotating frame experiment proved to be an efficient method of the slice selection.

The selected slice is generally narrower for the SI, procedure compared with ADRF. This is a direct consequence of the quasi-Gaussian cross-polarizatiom spectrum as comparad with the quasi-exponential cross-polarization spectrum in the ADRF case.

The width of selected slice becomes smaller as Ifartmann-JIalm mismatch increases and is becoming bigger for stronger contact pulse strength in the case of the ADRF cooling procedure.

It was shown that in the case of the SL-cooling procedure for some values of the experimental parameters a magic-slice can occur in the sample. $\Lambda$ polarization 
transfer process with a limited number of coupled spins takes place for this position and the shape of the selected slice should be locally modified. The presence of the magic slice in the sample region can be avoided by a proper selection of contact pulse strength, field gradient and sample dimension.

For a heterogeneous sample the spatial distribution of homonuclear and heteronuclear dipolar interactions and rotating frame relaxation rates will induce local variation in the width and slice profile. These cffects can be evaluated using the presented theory.

These results have implications not only for volume localized NMR spectroscopy but also for the NMIR imaging of magnetically dilute nuclei in solids.

\section{Acknowledgments}

We thank Professor Rainer Kimmich for valuable discussions.

\section{References}

[1] P. Mansficld, P.K. Grennel, Phys. Rev. B 12, 3618 (1975).

[2] D.G. Cory, J.B. Miller, R. Tenner, A.N. Garroway, Mol. Phys. 70, 331 (1990).

[3] W.I. Veeman, D.G. Cory, Adv. Magn. Reson. 13, 435 (1989).

[4] F. De Luca, B. Maraviglia, J. Magn. Reson. 67, 169 (1986).

[5] F. De Luca, C. Muccetelli, B.C. De Simone, B. Maraviglia, J. Magn. Reson. 69, 496 (1986).

[6] D.G. Cory, A.M. Reichwein, J.W.M. Van Os, W.S. Veeman, Chem. Phys. Lett. 143, 467 (1988).

[7] II.M. Cho, C.J. Lee, D.N. Skykind, D.P. Wcilckamp, Phys. Rev. Lelt. 55, 1923 (1985).

[8] I.B. Miller, A.N. Garroway, J. Magn. Reson. 82, 529 (1989).

[9] A.N. Garroway, J. Baum, M.G. Murowitz, A. P’iles, J. Magn. Reson. 60, 337 (1984).

[10] E. Günter, B. Blümich, H.W. Spics, Mol. Phys. 71, 147 (1990).

[11] S. Emid, J.H.N. Creyghton, Physica B 128, 81 (1985).

[12] P.J. McDonald, J.J. Attard, D.G. Taylor, J. Magn. Reson. 72, 224 (1987).

[13] D.E. Demco, S. Ilafuer, R. Kimmich, J. Magn. Reson. 94, 333 (1991).

[14] E. Rommel, S. Ilafner, R. Kimmich, J. Magn. Reson. 86, 268 (1990).

[15] D.E. Demco, S. IIafner, R. Kimmich, J. Magn. Reson. 96, 307 (1992).

[16] S. Matsui, K. Kose, T. Inonye, J. Magn. Reson. 88, 186 (1990).

[17] E. Rommel, P. Nickel, R. Kimmich, D. Pusiol, J. M/agn. Reson. 01, 630 (1991).

[18] N.H. Szevereny, G. Maciel, J. Magn. Reson. 60, 160 (1981).

[19] G.E. Maciel, M.F. Davis, J. Magn. Reson. 64, 356 (1985).

[20] D.G. Corry, W.S. Vccman, J. Phys. E, Sci. Instrum. 22, 180 (1989).

[21] D.E. Demco, R. Kimmich, Book of abstracts, 26th Congres Ampere on Magnetic Resonance, Eds. A. Anagnostopoulas, F. Milia, A. Sionpopoulos, Athens 1992.

[22] S. Hafner, D.E. Demco, R. Kïmmich, Chem. Phys. Lett. 187, 53 (1991). 
[23] M. Melıring, Principles of Jigh Resolution NMR in Solids, Springer-Verlag, Berlin 1983.

[24] R.R. Ernst, G. Bodenhausen, A. Wokaun, Principles of Nuclcar Magnelic Resonance in One and Two Dimensions, Clarendom Press, Oxford 1987.

[25] P. Mansficld, P.G. Morris, NAR Imaging in Biomedicine, Academic Press, New York 1982.

[26] D.I. Iloult, J. Magn. Reson. 38, 389 (1980).

[27] L. Kyle IIedges, D.I. IIoult, J. Magn. Reson. 79, 391 (1988).

[28] D.E. Demco, R. Kïimmich, S. IIafuer, II.W. Weber, J. Magn. Reson. 94, 317 (1991).

[29] D.E. Demco, J. Tegenfeldt, T.S. Waugh, Phys. Rev. B 11, 4133 (1975).

[30] H.T. Stokes, D.C. Nilion, Phys. Rev. B 15, 1271 (1977).

[31] M.II. Levitt, D. Suter, R.R. Erust, J. Chem. Phys. 84, 1243 (1986).

[32] T. Jecner, P. Brockacrt, Phys. Rev. 157, 232 (1967).

[33] A. Pines, M.G. Gibby, T.S. Wangh, J. Chem. Phys. 59, 569 (1973).

[34] L. Müller, A. Kimmar, T. Banmanu, R.R. Erust, Phys. Rev. Lett. 32, 1402 (1974).

[35] R.K. Ilester, T.L. Ackerman, V.R. Cross, T.S. Waugh, Phys. Rev. Lett. 34, 993 (1975).

[36] S.R. Hartmanı, E.L. IIalıı, Phys. Rev. 128, 2042 (1962).

[37] D.V. Lang, P.R. Morgan, Phys. Rev. B 1, 53 (1970).

[38] A. Pines, T.W. Shattuck, J. Chem. Phys. 61, 1255 (1974).

[39] D.A. McArthur, E.L. IIahu, R.E. Walstedt, Phys. Rev. 188, 609 (1969).

[40] S. Hafuer, privalc communication.

[41] E. Rommel, R. Kimmich, J. Magn. Rcson. 83, 299 (1989).

[42] S. Hafuer, E. Rommel, R. Iïimuich, J. Magn. Reson. 88, 449 (1990).

[4.3] M. Goldman, Spin Temperalure and Nuclear M/agnelic Resonance in Solids, Oxford U.P., London 1970. 